\title{
TROPHIC LEVEL \& EVOLUTION IN PALEOZOIC GASTROPODS
}

ALLMON, Warren D., Dept. of Geology, University of South Florida, Tampa, FL 33620; ERWIN, Douglas H., Dept. of Paleobiology, Smithsonian Institution, Washington, DC 20560; LINSLEY, Robert M., Geology Dept. Colgate University, Hamilton, NY 13346; MORRIS*, Paul J., Museum of Comparative Zoology, Harvard University, Cambridge, MA 02138.

Although trophic position or level is one of the most basic aspects of a benthic marine species' ecology, its evolutionary significance remains obscure. Gastropods offer a suitable model for examining the relationship between trophic level and evolution since they exhibit a wide variety of trophic strategies and their mode of life is often reflected in their shell form. We examined 196 genera of Paleozoic gastropods $(\approx 1 / 3$ of known genera) for which first appearance and last appearance could be specified to stage level and for which trophic strategy could be inferred with a reasonable degree of confidence. We classified these genera into four trophic categories on the basis of shell characters relating to locomotion and clamping. These trophic categories are: Suspension feeders, Grazers on firm substrata, Soft substrate Grazers/Detritivores, and Carnivores. Suspension feeders are the most unambiguously recognizable category, marked by clear indicators of a sessile mode of life such as a radial apertures and planispiral shell forms. Our central observation from these data is that suspension feeders have shorter generic longevities than the other three trophic groups. This pattern is robust to a variety of methods of analysis. The mean generic longevity of the suspension feeders is $15 \mathrm{MY}$ less than the other trophic categories. Cumulative frequency of genera within trophic categories versus log duration shows suspension feeders to be statisticaly significantly shorter lived than the other three trophic categories. The other three categories are not distinguishable. This pattern is unchanged by the removal of taxa dying out at mass extinctions. Suspension feeders have lower origination rates and higher extinction rates than the other trophic classes. This is not a taxonomic artifact produced by ornamentation and the number of characters available. This background pattern is also present in the end Ordovician and Late Devonian mass extinctions. Suspension feeders loose about half their genera in these extinctions, the two classes of grazers loose about $1 / 3$ of their genera, and the carnivores suffer almost no extinctions. Suspension feeding appears to carry a significant evolutionary detriment in both mass extinctions and background times. This may be reflected in the change in trophic distribution of gastropods from the Ordovician to the Recent. The end Permian extinction shows a different pattern of selectivity; detritivores suffer the least. 